\title{
Expression of multidrug resistance proteins in invasive ductal carcinoma of the breast
}

\author{
WEIQUAN LI and MAOMIN SONG
}

Department of General Surgery, Beijing Tiantan Hospital, Capital University of Medical Sciences, Beijing 100050, P.R. China

Received December 27, 2013; Accepted July 8, 2014

DOI: $10.3892 / 01.2014 .2435$

\begin{abstract}
Chemotherapy is commonly used for the treatment of breast cancer. However, the resistance to chemotherapeutic agents, often mediated by multidrug resistance (MDR) mechanisms, is a common occurrence. The present study examined the expression of several MDR-related proteins (MRPs) in invasive ductal carcinoma (IDC) of the breast, and assessed their association with clinicopathological variables and their prognostic significance. In addition, immunohistochemistry was used to measure the expression of MRP, p-glycoprotein (P-gp), topoisomerase $2 \alpha$ (Topo $2 \alpha$ ), thymidylate synthase (TS) and glutathione- $S$-transferase $\pi$ (GST- $\pi$ ) in 156 resected IDCs of the breast. Pearson's $\chi^{2}$ test and Spearman's correlation coefficient were used to analyze the association between MDR protein expression and several clinicopathological variables. The association between each of the five MDR proteins was also examined. Furthermore, Kaplan-Meier analysis and Cox regression modeling were used to assess overall survival. The expression of MRP, P-gp, Topo $2 \alpha$, TS and GST- $\pi$ was detected in $20.5 \%$ (32/156), 25.0\% (39/156), 84.0\% (131/156), $41.7 \%(65 / 156)$ and $41.0 \%(64 / 156)$ of cases examined, respectively. No correlation was identified between MRP and Topo- $2 \alpha$ and the clinicopathological variables examined. By contrast, P-gp $\left(\chi^{2}=20.226 ; \mathrm{P}<0.0001\right)$ and GST- $\pi\left(\chi^{2}=35.032\right.$; $\mathrm{P}<0.0001)$ were found to positively correlate with tumor grade. In addition, staining for TS was associated with axillary lymph node metastasis $\left(\chi^{2}=42.281 ; \mathrm{P}<0.0001\right)$. The expression levels of P-gp and GST- $\pi$ were found to be significantly correlated $(r=0.319 ; \mathrm{P}<0.0001)$. Furthermore, GST- $\pi$ expression was elevated in estrogen receptor-negative breast cancer $\left(\chi^{2}=17.407 ; \mathrm{P}<0.0001\right)$. Tumor histological grade, in addition to TS and GST- $\pi$ expression, were significant predictors of a poor survival outcome. TS and GST- $\pi$ are consequently useful
\end{abstract}

Correspondence to: Dr Weiquan Li, Department of General Surgery, Beijing Tiantan Hospital, Capital University of Medical Sciences, 6 Tiantanxili, Beijing 100050, P.R. China

E-mail:medscilb@126.com

Key words: breast neoplasm, multidrug resistance-related protein, topoisomerase $2 \alpha$, thymidylate synthase, glutathione-s-transferase $\pi$, multidrug resistance, immunohistochemistry, p-glycoprotein prognostic biomarkers in IDC, therefore, when establishing a personalized chemotherapeutic plan, the expression of MDR proteins must be considered.

\section{Introduction}

Invasive ductal carcinoma (IDC) of the breast is a malignant disease, which affects numerous females worldwide (1). Chemotherapy in the neoadjuvant and adjuvant settings is widely administered for the treatment of breast cancer (2). However, despite its success, resistance to chemotherapeutic agents is a common occurrence that is often attributable to mechanisms of multidrug resistance (MDR) $(3,4)$. Although proteins that mediate this resistance mechanism have been identified and have the potential to serve as biomarkers or prognostic indicators of outcome, the function of these MDR-related proteins (MRPs) in IDC of the breast has not been extensively investigated.

The critical proteins that mediate MDR in tumors include MRP, p-glycoprotein (P-gp), topoisomerase $2 \alpha$ (Topo $2 \alpha$ ), thymidylate synthase (TS) and glutathione- $S$-transferase $\pi$ $(\mathrm{GST}-\pi)$. A number of these proteins have been investigated in other tumor types, including esophageal, colorectal and endometrial cancer. In numerous instances, resistance is achieved by an increased efflux of chemotherapeutic agents out of the tumor. Occasionally, this is an acquired problem, as while certain tumors are initially responsive, they become resistant following prolonged treatment. In other cases, tumors fail to respond to therapy at all, a mechanism known as de novo resistance (5).

The function of these MRPs in IDC of the breast has not been extensively investigated. Notably, the expression of MRP, P-gp, Topo $2 \alpha$, TS and GST- $\pi$ exhibit the potential to serve as biomarkers for the disease and have prognostic significance. The aim of this study was to examine the expression of MRP, P-gp, Topo $2 \alpha$, TS and GST- $\pi$ in breast IDC, and assess their association with clinicopathological variables, as well as their prognostic significance. The results may aid clinicians in the design of unique treatment regimens for each individual patient.

\section{Materials and methods}

Patients and specimens. Samples were obtained from patients with IDC of the breast who underwent primary surgery at 
Beijing Tiantan Hospital, Capital University of Medical Sciences (Beijing, China) between 2005 and 2007.

Prior to patient enrolment, the expression of MRP, P-gp, Topo $2 \alpha$, TS, GST- $\pi$, ER, PR, HER2 and pP53 was analyzed by staining the excised tumor tissue. In total, samples from 156 female patients were analyzed. The patient age ranged between 32 and 75 years (median age, 52 years). No distant metastases were detected in any patients during pre-operative examination. Lumpectomy and axillary dissection was performed in 20 cases, radical mastectomy in 24 cases and modified radical mastectomy in 112 cases. Immunohistochemical staining was performed at Beijing Tiantan Hospital. The clinicopathological data are shown in Table I.

The follow-up period consisted of the time from the first day following surgery until December 2012. Survival time was calculated from the first day following surgery until mortality or the last follow-up. The study was approved by the ethics committee of Beijing Tiantan Hospital Affiliated to Capital Medical University (Beijing, China). All patients provided written informed consent.

Immunohistological analysis. All tumor tissues were fixed in neutral buffered $4 \%$ formaldehyde and embedded in paraffin. Immunohistochemical staining was performed using an avidin-biotin peroxidase system (SP-9000 kit; Zhongshan Goldenbridge Biotechnology Co., Ltd., Beijing, China). All primary antibodies and reagents were purchased from Beijing Zhongshan Goldenbridge Biotechnology Co., Ltd. The following monoclonal antibodies were used: MRP (OCRL-1), P-gp (C494), Topo2 $\alpha$ (3F6), TS (TS106), GST- $\pi$ (LW29), ER (1D5), PR (1A6), HER2 (CB11) and p53 (DO7). Antigen retrieval for all proteins, with the exception of GST- $\pi$, was performed in citrate buffer ( $\mathrm{pH}$ 6.0) by autoclaving for $180 \mathrm{sec}$ at $100^{\circ} \mathrm{C}$. Staining was performed using the LabVision Autostainer360 System (Maixin. Bio. Co.Ltd., Fuzhou, China).

Positive staining of the tumor cells was determined by the appearance of a brown-yellow color. Protein staining scores were defined as follows: 0 , negative or $<10 \%$ of tumor cells stained positive; $+1,10-25 \%$ of cells stained positive; +2 , $26-75 \%$ of cells stained positive; and $+3,>75 \%$ of cells stained positive. HER-2 overexpression was scored based on the degree of membrane staining according to the manufacturer's instructions for the HercepTest (6). The following parameters were applied for the assessment of HER-2 expression: 0, no membrane staining or membrane staining in $<10 \%$ of tumor cells; $1+$, faint/barely perceptible partial membrane staining in $>10 \%$ of tumor cells; $2+$, weak to moderate staining of the entire membrane in $>10 \%$ of tumor cells; and 3+, marked staining of the entire membrane in $>10 \%$ of tumor cells. A score of either 0 or $1+$ was considered negative and scores of $2+$ and $3+$ were considered positive for HER2 overexpression. For each sample, $\geq 10$ fields (magnification, $x 200$ ) were randomly selected for analysis, whereby $>500$ positive cells were counted, and the average was calculated. Scores were assigned independently by two different pathologists. In the case of a discordant result, additional fields were counted and analyzed.

Statistical analysis. Statistical analyses were carried out using SPSS 19.0 software (SPSS Inc., Chicago, IL, USA). Pearson's $\chi^{2}$ test and Spearman's correlation coefficient were used to analyze the association between MDR protein expression and clinicopathological variables, as well as ER, PR, HER2 and p53 status. Similar calculations were performed to assess the association between the five MDR proteins analyzed in the study. Survival analysis was used to determine prognostic significance using Kaplan-Meier analysis and the Cox regression model. $\mathrm{P}<0.05$ was considered to indicate a statistically significant difference.

\section{Results}

Patient characteristics. A total of 23 (14.7\%) cases exhibited stage I disease, 97 cases (62.2\%) exhibited stage II disease and 36 cases (23.1\%) presented with stage III. The median follow-up time was 61 months (range, 7-94 months). No patients were lost to follow-up. A total of 132 patients (84.6\%) received adjuvant chemotherapy, including anthracycline-based compounds (111 cases in total; 98 cases received anthracycline drugs + cyclophosphamide + fluorouracil, and 13 cases received anthracyclines + paclitaxel) or a cyclophosphamide + methotrexate + fluorouracil-based regimen for 4-8 cycles (21 cases). Additionally, 33 cases (21.2\%) received adjuvant radiotherapy (60Co or linear accelerator) at a dose $50 \mathrm{~Gy}$, or $60 \mathrm{~Gy}$ for breast-conserving surgery. A total of 46 cases (29.5\%) exhibited recurrent metastasis. Of these, 19 cases exhibited metastasis to the lung, 16 cases exhibited liver metastasis, 9 cases exhibited bone metastasis and two cases exhibited brain metastasis. A total of 43 mortalities occurred, including five mortalities due to non-cancer-associated causes, such as cardiovascular disease (Table I).

Association between MDR protein expression and clinicopathological variables, including HER-2, ER, PR and p53 status. The expression of MRP, P-gp, Topo $2 \alpha$, TS and GST- $\pi$ was detected in $20.5 \%$ (32/156), $25.0 \%$ (39/156), $84.0 \%(131 / 156), 41.7 \%(65 / 156)$ and $41.0 \%(64 / 156)$ of cases examined, respectively. Representative staining for each of the aforementioned proteins are shown in Fig. 1. Pearson $\chi^{2}$ analysis revealed that MRP and Topo2 $\alpha$ protein expression did not correlate with patient age, tumor size, axillary lymph node metastasis, histological grade, HER-2 overexpression or expression status of ER, PR and p53. P-gp expression was significantly higher in grade III tumors compared with grade I $\left(\chi^{2}=16.060 ; \mathrm{P}<0.001\right)$ and grade II $\left(\chi^{2}=13.563 ; \mathrm{P}<0.001\right)$ tumors. No significant difference in GST- $\pi$ staining was identified between grade I and II tumors $\left(\chi^{2}=2.492 ; \mathrm{P}=0.114\right)$, however, there was a significant difference between tumors of grades I and III $\left(\chi^{2}=16.001 ; \mathrm{P}<0.001\right)$ and II and III $\left(\chi^{2}=34.998\right.$; $\mathrm{P}<0.001)$. GST- $\pi$ expression was highest in grade III IDC breast tissue. GST- $\pi$ expression was also increased in ER-negative tumors $\left(65.3 \% ; \chi^{2}=17.407 ; \mathrm{P}<0.001\right)$ with a Spearman's correlation coefficient of $-0.437(\mathrm{P}<0.001)$. TS expression $(74.6 \%$; $44 / 59$ cases) was increased in breast cancer cases with axillary lymph node metastasis $\left(\chi^{2}=42.281 ; \mathrm{P}<0.001\right)$ (Table I).

Association between the expression of MRP, TS, Topo $2 \alpha$, $P$-gp and GST- $\pi$ proteins. Pearson's $\chi^{2}$ test was performed to examine the associations between the five MRPs. No significant correlation was identified between MRP protein expression and 


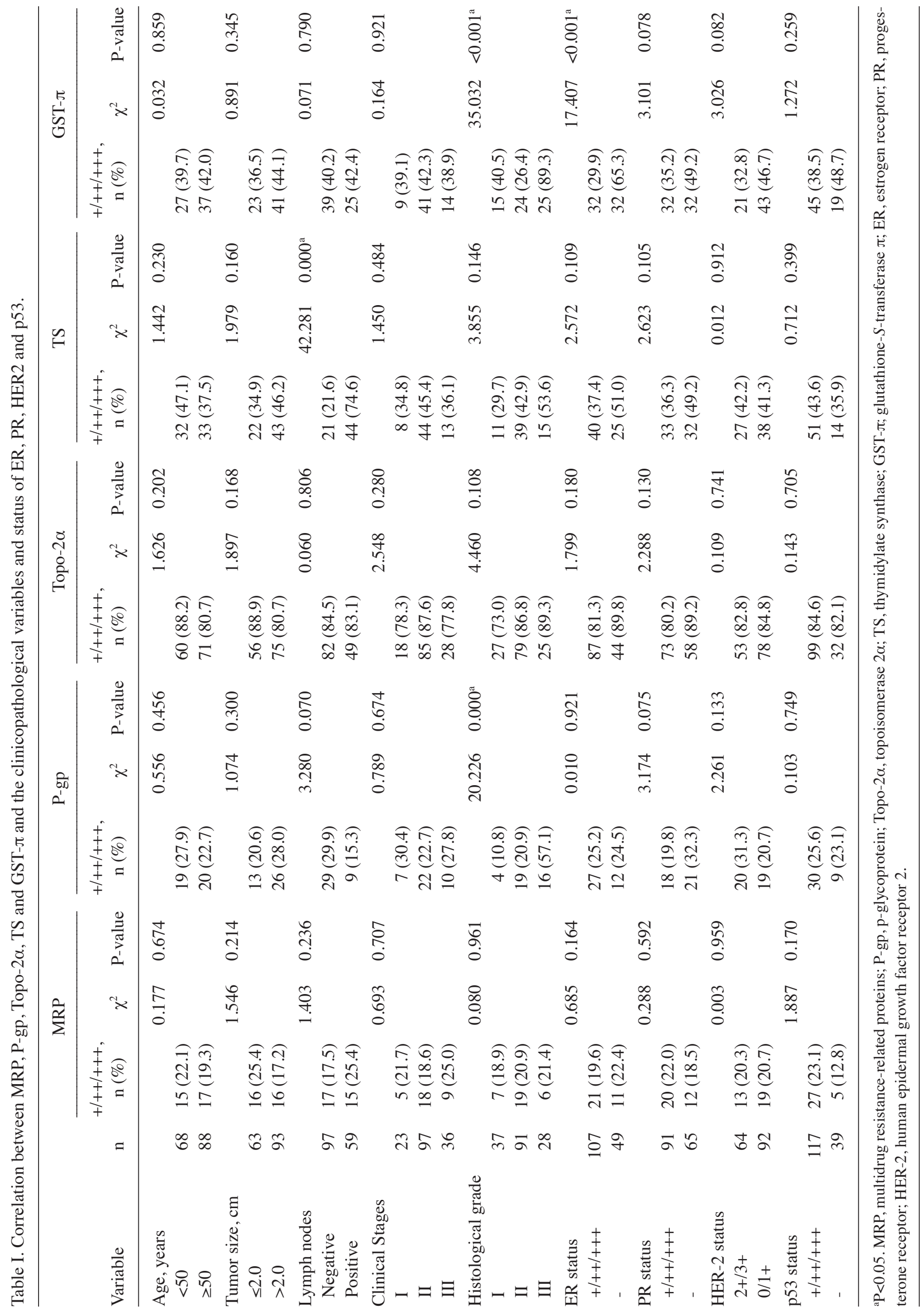


Table II. Cox univariate and multivariate analysis of five-year overall survival on MDR proteins and clinicopathological variables.

\begin{tabular}{|c|c|c|c|c|}
\hline \multirow{2}{*}{$\begin{array}{l}\text { MDR proteins and } \\
\text { clinicopathological variables }\end{array}$} & \multicolumn{2}{|c|}{ Univariate } & \multicolumn{2}{|c|}{ Multivariate } \\
\hline & HR $(95 \%$ CI $)$ & $\mathrm{P}$-value & HR $(95 \%$ CI $)$ & P-value \\
\hline MRP & $1.023(0.739-1.865)$ & 0.905 & $1.281(0.806-2.036)$ & 0.295 \\
\hline TS & $1.634(0.988-2.161)$ & $0.002^{\mathrm{a}}$ & $1.481(1.070-2.048)$ & $0.018^{\mathrm{a}}$ \\
\hline Торо $2 \alpha$ & $1.291(0.809-2.059)$ & 0.284 & $1.557(0.949-2.555)$ & 0.080 \\
\hline P-gp & $1.032(0.845-1.096)$ & 0.855 & $0.753(0.506-1.120)$ & 0.161 \\
\hline GST $-\pi$ & $1.683(0.917-2.325)$ & $0.001^{\mathrm{a}}$ & $1.853(1.284-2.674)$ & $0.001^{\mathrm{a}}$ \\
\hline Age & $0.976(0.872-1.085)$ & 0.115 & $1.016(0.966-1.069)$ & 0.538 \\
\hline Menstrual & $1.789(0.645-7.016)$ & 0.060 & $1.862(0.551-6.295)$ & 0.317 \\
\hline Tumor size & $1.002(0.502-1.592)$ & 0.995 & $0.959(0.482-1.908)$ & 0.905 \\
\hline Lymph node & $1.660(0.635-3.194)$ & 0.098 & $1.528(0.719-3.243)$ & 0.271 \\
\hline Histological grade & $3.471(1.122-4.125)$ & $<0.001^{\mathrm{a}}$ & $3.089(1.819-5.243)$ & $<0.001^{\mathrm{a}}$ \\
\hline
\end{tabular}

${ }^{a} \mathrm{P}<0.05$ was considered to indicate a statistically significant difference. MDR, multidrug resistance; TS, thymidylate synthase; $\mathrm{P}$-gp, $\mathrm{p}$-glycoprotein; Topo $2 \alpha$, topoisomerase $2 \alpha$; GST- $\pi$, glutathione- $S$-transferase $\pi$; CI, confidence interval.

the expression of TS $\left(\chi^{2}=3.523 ; \mathrm{P}=0.061\right)$, Topo2 $\alpha\left(\chi^{2}=2.409\right.$; $\mathrm{P}=0.121), \mathrm{P}-\mathrm{gp}\left(\chi^{2}=3.355 ; \mathrm{P}=0.067\right)$ or GST- $\pi\left(\chi^{2}=2.769\right.$; $\mathrm{P}=0.096)$. Furthermore, no significant association was identified between TS expression and the expression of Topo2 $\alpha\left(\chi^{2}=1.308\right.$; $\mathrm{P}=0.253), \mathrm{P}-\mathrm{gp}\left(\chi^{2}=1.064 ; \mathrm{P}=0.302\right)$ or GST- $\pi\left(\chi^{2}=2.047\right.$; $\mathrm{P}=0.153)$. Similarly, no significant correlation was identified between Topo $2 \alpha$ and GST- $\pi\left(\chi^{2}=0.599 ; \mathrm{P}=0.439\right)$ or P-gp $\left(\chi^{2}=0.397 ; \mathrm{P}=0.529\right)$. However, a significant positive correlation was identified between P-gp and GST- $\pi\left(\chi^{2}=20.348 ; \mathrm{P}<0.001\right)$ with a Spearman's correlation coefficient of $0.319(\mathrm{P}<0.001)$.

TS and GST- $\pi$ expression are associated with poor overall survival. Positive staining of MRP, P-gp and Topo2 $\alpha$ were not found to significantly correlate with changes in overall survival (Fig. 2). Kaplan-Meier survival analyses revealed that patients with tumor specimens that stained positive for TS expression exhibited significantly poorer overall survival rates than patients with TS-negative tumors ( $\mathrm{P}=0.001$; Fig. 2B). Similarly, patients with GST- $\pi$-positive tumors had a poorer overall survival rate compared with patients with GST- $\pi$-negative breast tumors ( $\mathrm{P}=0.001$; Fig. 2C). TS and GST- $\pi$ were then examined to determine whether they represent independent prognostic factors in the disease. As shown in Table II, Cox univariate analysis revealed that positive staining for TS or GST- $\pi$ was associated with a significantly increased risk of mortality in breast carcinoma patients (TS, $\mathrm{P}=0.002$; GST- $\pi, \mathrm{P}=0.001$ ). These factors were also positively associated with histological grade $(\mathrm{P}<0.001)$. Cox multivariate analysis indicated that TS and GST $-\pi$ were independent prognostic factors $(\mathrm{P}=0.018$ and $\mathrm{P}=0.001$, respectively) and that tumor grade was a predictor of a poor survival outcome $(\mathrm{P}<0.001)$.

\section{Discussion}

MDR is a common mechanism by which tumor cells become resistant to numerous chemotherapeutic agents. While this MDR response is multi-faceted, it typically involves the upregulation of several key proteins that promote the efflux of drugs out of tumor cells, decreasing their biological efficacy. In the present study, the expression of several key MDR-associated proteins, including MRP, P-gp, Topo2 $\alpha$, TS and GST- $\pi$, was investigated in IDC of the breast. The study analyzed the expression of these factors together with several clinicopathological variables and overall survival. We hypothesized that the expression of these factors may be useful as biomarkers of the disease and may explain the occurrence of chemotherapy resistance.

The expression of one of the proteins examined, P-gp, was significantly higher in grade III tumors compared with grade I and II tumors. Previous studies have demonstrated that this protein promotes the efflux of a number of anticancer drugs, including anthracyclines, vinca alkaloids, taxanes, epipodophyllotoxins and doxorubicin, out of tumor cells $(7,8)$. P-gp is also involved in the secretion of anticancer agents into bile, urine and the intestinal lumen, which markedly affects the pharmacokinetic properties and bioavailability of therapeutically administered compounds (9). Linn et al (10) evaluated P-gp expression in 92 primary and 12 metastatic breast cancers and found that P-gp expression was associated with a poor prognosis. In another study, metastatic breast cancer patients negative for $\mathrm{P}$-gp expression $(\mathrm{P}=0.06)$ exhibited a longer progression-free survival time following docetaxel treatment compared with patients exhibiting P-gp-positive tumors (11). In the present study, P-gp and GST- $\pi$ expression were found to be positively correlated $(\mathrm{r}=0.319 ; \mathrm{P}<0.0001)$. Additionally, the expression of the two proteins was elevated in grade III tumors. Consistent with the findings of the present study, Cui et al (12) identified a positive correlation $(\mathrm{r}=0.429 ; \mathrm{P}<0.01)$ between P-gp and GST- $\pi$ in 76 breast cancer patients prior to treatment. Furthermore, another study investigated the correlation between the expression of P-gp, GST and metallothioneins (MTs) and the response to various chemotherapy regimens in triple--negative (ER-, PR- and HER2-negative) breast cancer patients (13). The chemotherapy-treated groups demonstrated improved three-year relapse-free survival rates $(\mathrm{P}<0.05)$, which were associated with the expression of P-gp, GST and 
A

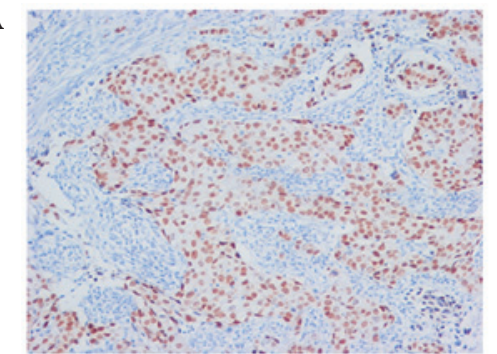

D

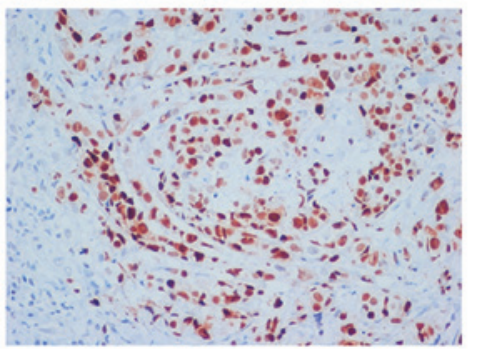

G

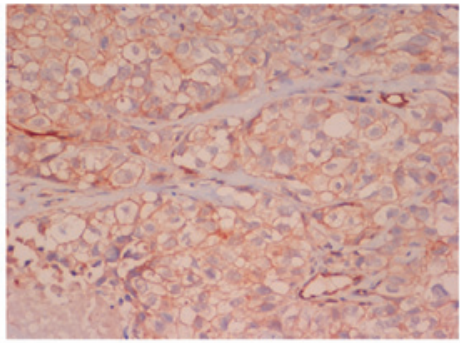

B

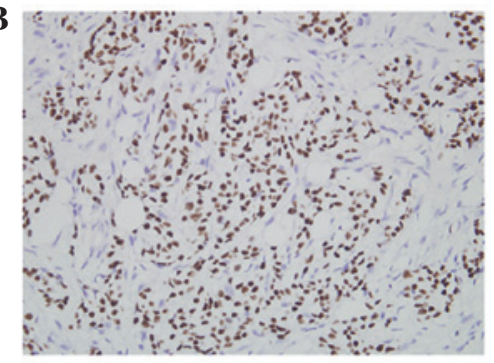

$\mathbf{E}$

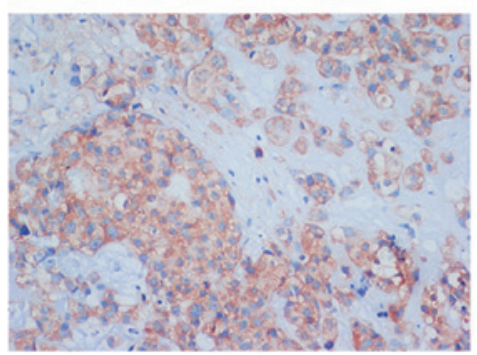

H

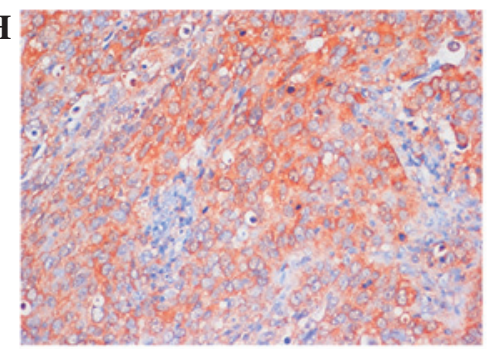

C

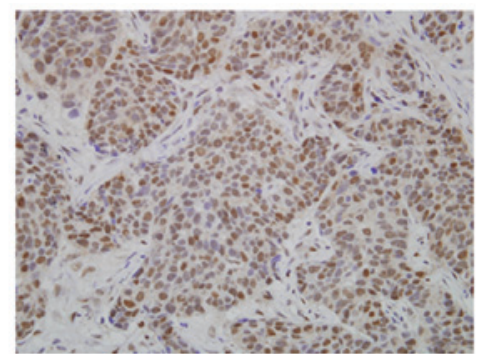

F

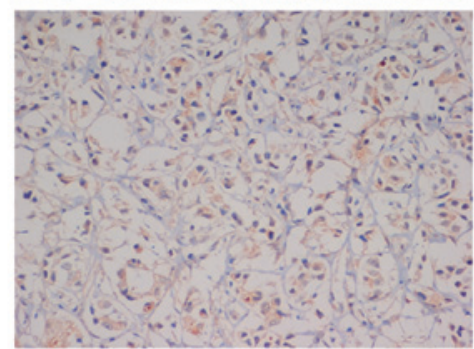

I

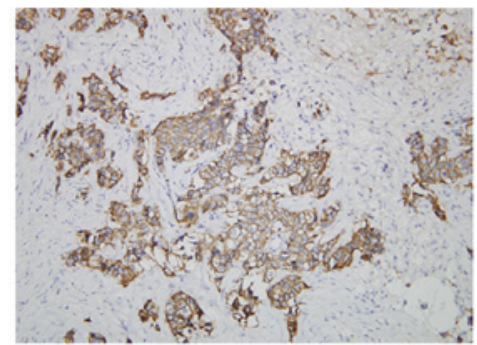

Figure 1. Positive immunohistochemical staining of (A) ER, (B) PR, (C) p53, (D) Topo2 $\alpha$, (E) GST- $\pi$, (F) TS, (G) P-gp and (H) MRP, and (I) HER-2 overexpression in invasive ductal carcinoma of breast (streptomycin avidin-peroxidase, magnification, $\mathrm{x} 200$ ). ER, estrogen receptor; PR, progesterone receptor; Topo $2 \alpha$, topoisomerase $2 \alpha$; GST- $\pi$, glutathione-S-transferase; TS, thymidylate synthase; P-gp, p-glycoprotein; MRP, multidrug resistance-related protein; HER-2, human epidermal growth factor receptor 2.
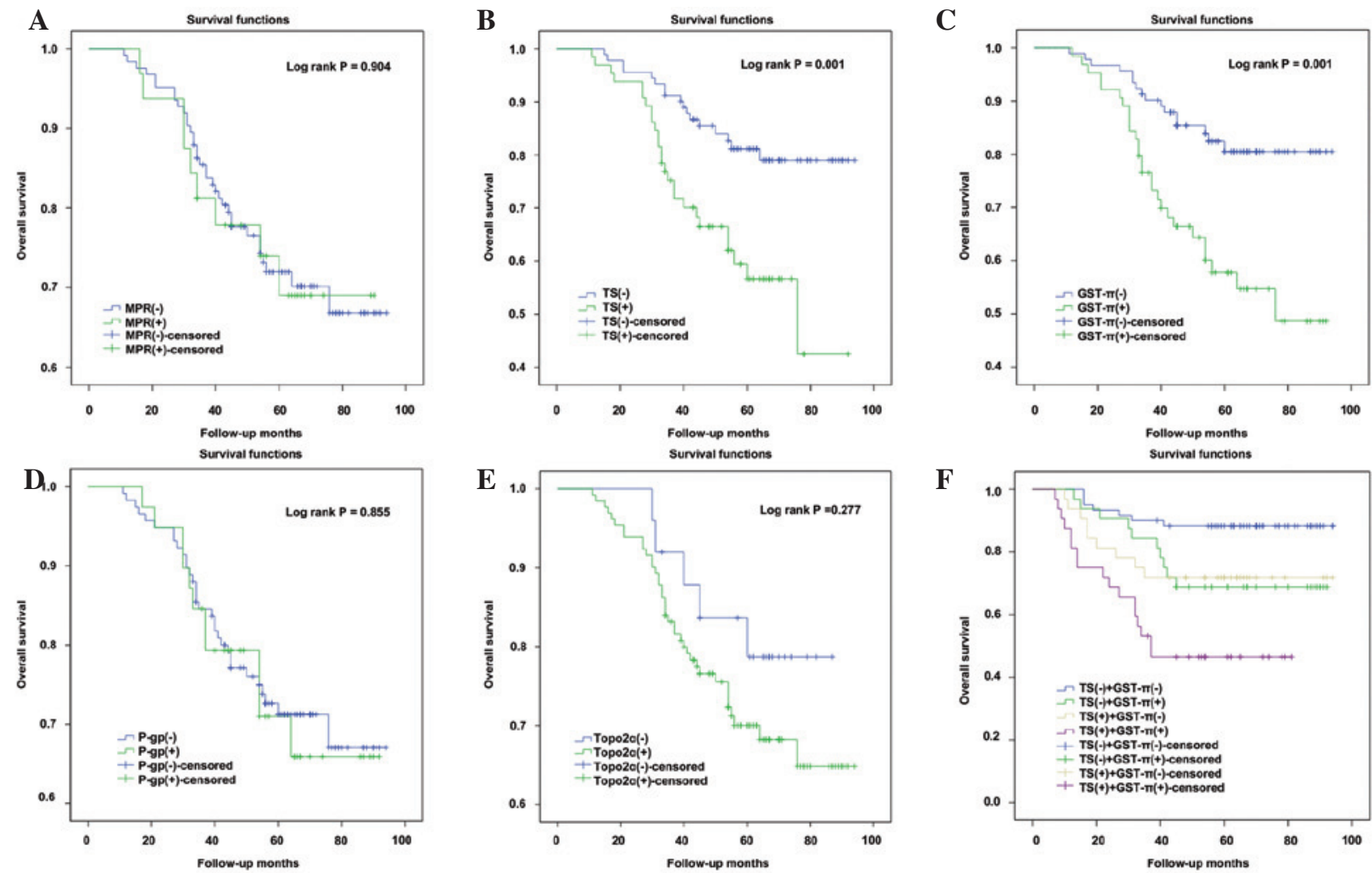

Figure 2. Analysis of the correlations between the five multidrug resistance proteins (A) MRP (B) TS (C) GST- $\pi$ (D) P-gp (E) Topo2 $\alpha$ and (F) combined expression of TS and GST- $\pi$ and overall survival. Patients with positive TS and GST- $\pi$ expression exhibited a significantly shorter overall survival time than patients with negative expression for the two proteins. MRP, multidrug resistance-related protein; TS, thymidylate synthase; GST- $\pi$, glutathione-S-transferase; P-gp, p-glycoprotein; Topo2 $\alpha$, topoisomerse $2 \alpha$. 
MT. These results, in addition to the results of the present study, indicate a function for these MRPs in the outcome of breast cancer and its response to chemotherapy.

As aforementioned, GST- $\pi$ expression was highest in grade III IDC breast tumors and also increased in ER-negative disease. Notably, patients with tumors staining positive for GST- $\pi$ were also found to exhibit poorer overall survival rates than patients with GST- $\pi$-negative tumors. Thus, the results of this study indicated that GST- $\pi$ is associated with a worse prognosis and may potentially drive disease progression. These findings are supported by previous studies. For example, resistance against drugs and environmental insults is conferred by the glutathione metabolic pathway (4). The GSTs are a family of enzymes involved in the metabolism of a broad range of xenobiotics, which have been shown to inactivate platinum drugs, doxorubicin, cyclophosphamide and etoposide $(14,15)$. In a previous study, the expression of GST- $\pi$ was investigated in 21 primary untreated human breast tumors (16) and in agreement with the findings of the present study, the mean expression of GST- $\pi$ in ER-negative tumors was found to be 5 -fold greater than the mean expression in ER-positive tumors. These findings were also consistent with another study examining 189 breast cancer cases (17). Overall, patients with ER-negative tumors may exhibit increased resistance to chemotherapeutic regimens due to increased GST- $\pi$ levels. The results of the present study indicate an association between ER and GST- $\pi$, which requires additional investigation in the future.

Topo2 is an essential nuclear DNA-binding enzyme that controls and modifies the topological states of DNA by combining nuclease, helicase and ligase activities (18). Topo2 $\alpha$ is a specific isoform that is located on chromosome 17q21 in close proximity to HER-2. The exact association between Topo $2 \alpha$ and HER-2 remains unclear. While certain studies have found no association between Topo $2 \alpha$ and HER 2 overexpression (19-21), other studies have reported that the increased expression of Topo $2 \alpha$ is associated with HER-2 amplification or overexpression $(22,23)$. To better define the clinical relevance of Topo $2 \alpha$ expression, larger prospective studies are required. In this study, positive staining for Topo $2 \alpha$ was identified in $84.0 \%$ (131/156) of the cases examined, indicating that Topo $2 \alpha$ expression may be significant in breast cancer. Notably, Mukherjee et al (24) revealed that the expression of Topo $2 \alpha$ prior to the administration of chemotherapy significantly correlated with the pathological complete response to neoadjuvant anthracycline treatment. Another study reported that ER is an independent predictive factor for pathological response to three different pre-operative chemotherapy regimens in primary breast tumors (25), however, the expression of PR, Topo2, P-gp, MRP and GST- $\pi$ were not predictive of the pathological response to the three treatment regimens.

TS is a folate-dependent enzyme involved in pyrimidine synthesis that is crucial for cellular proliferation and growth (26). TS also catalyzes the methylation of deoxyuridine monophosphate to deoxythymidine monophosphate, an essential precursor of DNA biosynthesis (27). In the present study, TS expression was found to be elevated in cases of invasive breast carcinoma with lymph node metastasis. Thus, these breast cancers are more aggressive and exhibit a poorer overall prognosis. Furthermore, a similar association between TS levels and prognosis has been reported in other tumor types, including colorectal, rectal and gastric cancers (28-30). TS overexpression is a biomarker of 5-fluorouracil (5-FU) resistance in human cancer cells (31). However, 5-FU combined with low-dose trichostatin A $(50 \mathrm{nmol} / \mathrm{l})$ has been shown to restore 5-FU-mediated cytotoxicity in 5-FU-resistant cancer cells in combination with the downregulation of TS protein expression (31). In another study of advanced-stage breast cancer patients, lower TS levels were associated with an improved response to the chemotherapy drug pemetrexed. Additionally, in a number of patients, continuous administration of pemetrexed has been found to decrease TS levels (32). Brandi et al (33) revealed that patients with low levels of TS and high levels of p53 responded better to docetaxel. Overall, these results indicate that low levels of TS may be associated with an improved breast cancer prognosis and response to chemotherapy administration.

In conclusion, the assessment of MDR protein expression in breast cancer may be a useful predictor of prognosis and the response to chemotherapy. Ultimately, this information may aid clinicians in the design of unique treatment regimens for each individual patient.

\section{References}

1. Liu H, Liu Y and Zhang JT: A new mechanism of drug resistance in breast cancer cells: fatty acid synthase overexpressionme-mediated palmitate overproduction. Mol Cancer Ther 7: 263-270.

2. Cance WG, Carey LA, Calvo BF, et al: Long-term outcome of neoadjuvant therapy for locally advanced breast carcinoma: effective clinical downstaging allows breast preservating and predicits outstanding local control and survival. Ann Surg 236: 295-303, 2002.

3. Sachelarie I, Grossbard ML, Chadha M, et al: Primary systemic therapy of breast cancer. Oncologist 11: 574-589, 2006.

4. LaPensee EW and Ben-Jonathan N: Novel roles of prolactin and estrogens in breast cancer: resistance to chemotherapy. Endocr Relat Cancer 17: R91-R107, 2010.

5. Coley HM: Mechanisms and strategies to overcome chemotherapy resistance in metastatic breast cancer. Cancer Treat Rev 34: 378-390, 2008.

6. Jacobs TW, Gown AM, Yaziji H, Barnes MJ and Schnitt SJ: Specificity of HercepTest in determining HER-2/neu status of breast cancers using the United States Food and Drug Administration-approved scoring system. J Clin Oncol 17: 1983-1987, 1999.

7. Gottesman MM, Fojo T and Bates SE: Multidrug resistance in cancer: role of ATP-dependent transporters. Nat Rev Cancer 2: 48-58, 2002.

8. Sekine I and Saijo N: Polymorphisms of metabolizing enzymes and transporter proteins involved in the clearance of anticancer agents. Ann Oncol 12: 1515-1525, 2001.

9. Orlowski S and Garrigos M: Multiple recognition of various amphiphilic molecules by the multidrug resistance P-glycoprotein: molecular mechanisms and pharmacological consequences coming from functional interactions between various drugs. Anticancer Res 19: 3109-3123, 1999.

10. Linn SC, Giaccone G, van Diest PJ, et al: Prognostic relevance of P-glycoprotein expression in breast cancer. Ann Oncol 6: 679-685, 1995.

11. Savas B, Bozcuk H, Özdo M, et al: Molecular and clinical parameters which determine the docetaxel response in metastatic breast cancer. J Clin Oncol 22: 9732, 2004.

12. Cui SD, Liu ZZ, Liu H, Li LF, Yang H and Li WL: The relationship between $99 \mathrm{mTc}-\mathrm{MIBI}$ scintimammography of breast cancer and multidrug-resistant proteins. Zhonghua Zhong Liu Za Zhi 27: 606-608, 2005 (In Chinese).

13. Chekhun VF, Zhylchuk VE, Lukyanova NY, Vorontsova AL and Kudryavets YI: Expression of drug resistance proteins in triple-receptor-negative tumors as the basis of individualized therapy of the breast cancer patients. Exp Oncol 31: 123-124, 2009.

14. Jakoby WB: The glutathione S-transferases: a group of multifunctional detoxification proteins. Adv Enzymol Relat Areas Mol Biol 46: 383-414, 1978. 
15. Tew KD: Glutathione-associated enzymes in anticancer drug resistance. Cancer Res 54: 4313-4320, 1994.

16. Moscow JA, Townsend AJ, Goldsmith ME, et al: Isolation of the human anionic glutathione S-transferase cDNA and the relation of its gene expression to estrogen-receptor content in primary breast cancer. Proc Natl Acad Sci USA 85: 6518-6522, 1988.

17. Gilbert L, Elwood LJ, Merino M, et al: A pilot study of pi-class glutathione S-transferase expression in breast cancer: correlation with estrogen receptor expression and prognosis in node-negative breast cancer. J Clin Oncol 11: 49-58, 1993.

18. Colozza M, Azambuja E, Cardoso F, Sotiriou C, Larsimont D and Piccart MJ: Proliferative markers as prognostic and predictive tools in early breast cancer: where are we now? Ann Oncol 16 : $1723-1739,2005$

19. Coon JS, Marcus E, Gupta-Burt S, et al: Amplification and overexpression of topoisomerase IIalpha predict response to anthracycline-based therapy in locally advanced breast cancer. Clin Cancer Res 8: 1061-1067, 2002.

20. MacGrogan G, Rudolph P, Mascarel Id Id, et al: DNA topoisomerase IIalpha expression and the response to primary chemotherapy in breast cancer. Br J Cancer 89: 666-671, 2003.

21. Campiglio M, Somenzi G, Olgiati C, et al: Role of proliferation in HER2 status predicted response to doxorubicin. Int J Cancer 105 568-573, 2003

22. Depowski PL, Rosenthal SI, Brien TP, Stylos S, Johnson RL and Ross JS: Topoisomerase IIalpha expression in breast cancer: correlation with outcome variables. Mod Pathol 13: 542-547, 2000.

23. Rudolph P, Olsson H, Bonatz G, et al: Correlation between $\mathrm{p} 53$, c-erbB-2, and topoisomerase II alpha expression, DNA ploidy, hormonal receptor status and proliferation in 356 node-negative breast carcinomas: prognostic implications. J Pathol 187: 207-216, 1999.

24. Mukherjee A, Shehata M, Moseley P, Rakha E, Ellis I and Chan S: Topo2alpha protein expression predicts response to anthracycline combination neo-adjuvant chemotherapy in locally advanced primary breast cancer. Br J Cancer 103: 1794-1800, 2010 .
25. Wang L, Jiang Z, Sui M, Shen J, Xu C and Fan W: The potential biomarkers in predicting pathologic response of breast cancer to three different chemotherapy regimens: a case control study. BMC Cancer 9: 226, 2009.

26. Navalgund LG, Rossana C, Muench AJ and Johnson LF: Cell cycle regulation of thymidylate synthetase gene expression in cultured mouse fibroblasts. J Biol Chem 255: 7386-7390, 1980.

27. Carreras CW and Santi DV: The catalytic mechanism and structure of thymidylate synthase. Annu Rev Biochem 64: 721-762, 1995.

28. Aschele C, Debernardis D, Casazza S, et al: Immunohistochemical quantitation of thymidylate synthase expression in colorectal cancer metastases predicts for clinical outcome to fluorouracil-based chemotherapy. J Clin Oncol 17: 1760-1770, 1999.

29. Johnston PG, Fisher ER, Rockette HE, et al: The role of thymidylate synthase expression in prognosis and outcome of adjuvant chemotherapy in patients with rectal cancer. J Clin Oncol 12: 2640-2647, 1994.

30. Suda Y, Kuwashima Y, Tanaka Y, Uchida K and Akazawa S: Immunohistochemical detection of thymidylate synthase in advanced gastric cancer: a prognostic indicator in patients undergoing gastrectomy followed by adjuvant chemotherapy with 5-fluoropyrimidines. Anticancer Res 19: 805-810, 1999.

31. Lee JH, Park JH, Jung Y, et al: Histone deacetylase inhibitor enhances 5-fluorouracil cytotoxicity by down-regulating thymidylate synthase in human cancer cells. Mol Cancer Ther 5: 3085-3095, 2006

32. Gomez HL, Santillana SL, Vallejos CS, et al: A phase II trial of pemetrexed in advanced breast cancer: clinical response and association with molecular target expression. Clin Cancer Res 12: 832-838, 2006.

33. Brandi M, Calascibetta A, Cabibi D, et al: Relationship between thymidylate synthase expression and p53 levels with the treatment of cyclophosphamide, methotrexate, 5-fluorouracil chemotherapy (CMF) versus docetaxel (TXT) in locally advanced carcinoma of the breast. J Clin Oncol 24: 10546, 2006. 